CERN-TH.6991/93

FTUAM. 93/28

July, 1993

\title{
SOME GLOBAL ASPECTS OF DUALITY IN STRING THEORY
}

\author{
E. Alvarez ${ }^{*}$, L. Alvarez-Gaumé, J.L.F. Barbón ${ }^{* \star}$, Y. Lozano* \\ Theory Division, CERN \\ CH-1211 Geneva 23, Switzerland
}

\begin{abstract}
We explore some of the global aspects of duality transformations in String Theory and Field Theory. We analyze in some detail the equivalence of dual models corresponding to different topologies at the level of the partition function and in terms of the operator correspondence for abelian duality. We analyze the behavior of the cosmological constant under these transformations. We also explore several examples of non-abelian duality where the classical background interpretation can be maintained for the original and the dual theory. In particular, we construct a non-abelian dual of $S L(2, R)$ which turns out to be a three-dimensional black hole.
\end{abstract}

* Permanent address Departamento de Física Teórica, Universidad Autónoma de Madrid, Canto Blanco, Madrid, Spain

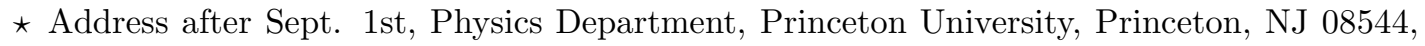
USA 


\section{INTRODUCTION}

Duality symmetry plays an important rôle in Statistical Mechanics (for a review and references to the literature see for instance [1]), in particular in the analysis of the phase diagram of spin systems. It can also be understood as a way to show the equivalence between two apparently different theories. On a lattice system described by a Hamiltonian $H\left(g_{i}\right)$ with coupling constants $g_{i}$ the duality transformation produces a new Hamiltonian $H^{*}\left(g_{i}^{*}\right)$ with coupling constants $g_{i}^{*}$ on the dual lattice. In this way one can often relate the strong coupling regime of $H(g)$ with the weak coupling regime of $H^{*}\left(g^{*}\right)$. In String Theory and Two-Dimensional Conformal Field Theory duality is an important tool to show the equivalence of different geometries and/or topologies and in determining some of the genuinely stringy implications on the structure of the low energy Quantum Field Theory limit. Duality symmetry was first described on the context of toroidal compactifications $[2,3]$. For the simplest case of a single compactified dimension of radius $R$, the entire physics of the interacting theory is left unchanged under the replacement $R \rightarrow \alpha^{\prime} / R$ provided one also transforms the dilaton field $\phi \rightarrow \phi-\log \left(R / \sqrt{\alpha^{\prime}}\right)$ [4]. This simple case can be generalized to arbitrary toroidal compactifications described by constant metric $g_{i j}$ and antisymmetric tensor $b_{i j}$ [5]. The generalization of the duality symmetry in this case becomes $(g+b) \rightarrow(g+b)^{-1}$ and $\phi \rightarrow \phi-\frac{1}{2} \log \operatorname{det}(g+b)$. In fact this transformation is an element of an infinite order discrete symmetry group for d-dimensional toroidal compactifications $O(d, d ; Z)[6]$. The symmetry was later extended to the case of non-flat conformal backgrounds in [7]. In Buscher's construction one starts with a manifold $M$ with metric $g_{i j}, i, j=0, \ldots d-1$, antisymmetric tensor $b_{i j}$ and dilaton field $\Phi\left(x_{i}\right)$. One requires the metric to admit at least one continuous isometry which leaves invariant the $\sigma$-model action constructed out of $(g, b, \phi)$. Choosing an adapted coordinate system $\left(x^{0}, x^{\alpha}\right)=\left(\theta, x^{\alpha}\right), \alpha=1, \ldots d-1$ where the isometry acts by translations of $\theta$, the change of $g, b, \phi$ is given by 


$$
\begin{aligned}
\tilde{g}_{00} & =1 / g_{00}, \quad \tilde{g}_{0 \alpha}=b_{0 \alpha} / g_{00}, \quad \tilde{g}_{\alpha \beta}=g_{\alpha \beta}-\frac{g_{0 \alpha} g_{0 \beta}-b_{0 \alpha} b_{0 \beta}}{g_{00}} \\
\tilde{b}_{0 \alpha} & =\frac{g_{0 \alpha}}{g_{00}}, \quad \tilde{b}_{\alpha \beta}=b_{0 \beta}-\frac{g_{0 \alpha} b_{0 \beta}-g_{0 \beta} b_{0 \alpha}}{g_{00}} \\
\tilde{\phi} & =\phi-\log g_{00} .
\end{aligned}
$$

The result is that for any continuous isometry of the metric which is a symmetry of the action one obtains the equivalence of two apparently very different non-linear $\sigma$-models. If $\mathrm{n}$ is the maximal number of commuting isometries, one obtains a duality group of the form $O(n, n ; Z)[8]$ (a rather thorough review of recent results in duality can be found in [9]). Duality symmetries are useful in determining important properties of the low-energy effective action, in particular in questions related to supersymmetry breaking and in the removal of flat directions from the potential [10]. Although the transformation (1.1) was originally obtained using a method apparently not compatible with general covariance, it is not difficult to modify the construction to eliminate this drawback. A particularly useful interpretation of (1.1) is in terms of the gauging of the isometry symmetry [11] with an auxiliary gauge field whose field strengh is required to vanish, although it will in general have non-trivial monodromy in non-spherical topologies. The analysis in [11] relates the two dual $d$-dimensional $\sigma$-models with an auxiliary $d+1$-dimensional $\sigma$-model which contains a left- and a right-handed chiral current associated to the original isometry. The original model and its dual are then obtained by gauging respectively the vector and axial vector combinations of chiral currents on the auxiliary theory. This presentation also clarified the relation between the conformal properties of the three models involved.

A further generalization of the general procedure in [11] was proposed in [12] in the case that the manifold $(M, g)$ has non-abelian isometries. This form of "duality" contains many striking differences with ordinary or abelian duality, and it is one of our purposes to explore some of its properties.

The outline of this paper is the following. In section two we present the general form of the duality transformation for compact abelian isometries. We use a 
manifestly covariant formalism and therefore we can control the global topological properties of the dual model. In particular we are interested in the case where the original manifold has a trivial fundamental group. Naively one would not expect to find any topology change in this case because the original model does not contain any winding states, and toroidal duality can be expressed as the exchange of winding and momentum states. We will see that generically the duality transformation changes the topology and/or the geometry of the manifold in a way largely independent of its fundamental group. In section three we study in detail a particular example (the $\sigma$-model on the three-sphere) as an illustrative case of a manifold without fundamental group whose topology changes to $S^{2} \times S^{1}$. We perform the duality transformation patch by patch and we explicitly reconstruct the topology of the dual manifold. Section four contains the explicit study of abelian duality in Wess-Zumino-Witten models where one can explicitly control the global topology rather easily. Section five presents the explicit map between operators of the original and dual theories (order/disorder mapping) for arbitrary curved backgrounds. In section six we make some remarks concerning the behavior of the cosmological constant under duality transformation and explore briefly some of its consequences. Finally in section seven we come to the study of non-abelian duality. We present a series of interesting examples based on manifolds with non-abelian isometry groups acting without fixed-points. The simplest example is provided by the $S U(2)$-nonlinear $\sigma$-model and as isometry group we take the left action of $S U(2)$ on itself. The dual model does not contain singularities, but it is non-compact (it is the Lie algebra of $S U(2)$ with a curved metric). Next we study the same procedure for the $S L(2, R) \sigma$-model. $S L(2, R)$ is geometrically anti-DeSitter space, and under non-abelian duality we obtain a three-dimensional anti-DeSitter black hole. In this case, although the isometries act without fixed points, the singularity appears as a consequence of the non-compactness of the isometry group. After some brief survey of other examples, we study some of the still unresolved questions in trying to construct the non-abelian duality transformation for higher genus Riemann surfaces, and the difficulties with the explicit construction of the operator mapping 
between the original model and its dual.

\section{GENERALLY COVARIANT DUALITY TRANSFORMATION}

In (1.1) we saw that the original construction of the duality transformation required a particular choice of coordinates. Since we are interested in the global topological properties of the dual theory $(\tilde{M}, \tilde{g}, \tilde{b})$, we would like to begin by writing the form of (1.1) in a general covariant form. This is all the more interesting in those cases we will consider later where $\pi_{1}(M)=0$. From a String Theory point of view there are only momentum modes and no winding modes, and thus we wish to clarify first of all what is the global topology of $\tilde{M}$ and the form of the operator mapping in this case. The way to proceed following the ideas in [11] is to gauge the isometry. With a general $b_{i j}$ field (Wess-Zumino-Witten term) we need to use the Noether procedure as made explicit in [13]. After gauging the isometry we impose the constraint that the gauge field should be flat. In this formalism going to adapted coordinates is a question of gauge fixing. However issues related to the resulting global topology and geometry can be addressed without recurring to a particular gauge choice, and we can always keep explicit general covariance.

Ignoring the dilaton, we consider the $\sigma$-model

$$
\begin{aligned}
S & =\frac{1}{2 \pi} \int d^{2} \sigma\left(g_{i j}+b_{i j}\right) \partial x^{i} \bar{\partial} x^{j}= \\
& =\frac{1}{8 \pi} \int g_{i j} \partial_{\mu} x^{i} \partial^{\mu} x^{j}+\frac{i}{8 \pi} \int b_{i j} d x^{i} \wedge d x^{j},
\end{aligned}
$$

the second term on the right-hand side is the Wess-Zumino term. If the world-sheet is a genus $g$ Riemann surface $\Sigma_{g}$, the Wess-Zumino term can be described in terms of an element of $H^{3}(M, R)$. If $\Sigma_{g}^{0}=$ interior of $\Sigma_{g}$, and $H$ is the selected 3 -form in $H^{3}(M, R)$, the Wess-Zumino-Witten term in the action is

$$
\Gamma=\frac{i}{8 \pi} \int_{\Sigma_{g}^{0}, \partial \Sigma_{g}^{0}=\Sigma_{g}} H
$$

We assume $H$ can be chosen in such a way that the ambiguity in $\Gamma$ due to different 
choices of $\Sigma_{g}^{0}$ is equal to $2 \pi i$ (integer), and the quantum theory is thus well-defined. Let $k^{i}$ be a Killing vector for the metric $g$ :

$$
\mathcal{L}_{k} g_{i j}=\nabla_{i} k_{j}+\nabla_{j} k_{i}=0
$$

Invariance of $S$ requires also

$$
\delta_{k} \Gamma=\frac{i}{8 \pi} \int_{\Sigma_{g}^{0}} \epsilon \mathcal{L}_{k} H=\frac{i}{8 \pi} \int_{\Sigma_{g}^{0}} \epsilon\left(d i_{k}+i_{k} d\right) H=\frac{i}{8 \pi} \epsilon \int_{\Sigma_{g}} i_{k} H=0,
$$

because $d H=0$, and $\left(i_{k} H\right)_{i j}=k^{l} H_{l i j}$, hence (2.4) vanishes if $i_{k} H=-d v$, for some 1-form $v$. In terms of $b,(H=d b$ locally $)$ this implies:

$$
\mathcal{L}_{k} b=d \omega, \quad \omega=i_{k} b-v
$$

The associated conservation law is:

$$
\begin{gathered}
\partial \bar{J}_{k}+\bar{\partial} J_{k}=0 \\
J_{k}=\left(k-i_{k} b+\omega\right)_{i} \partial x^{i}=(k-v)_{i} \partial x^{i} \equiv(k-v) \cdot \partial x \\
\bar{J}_{k}=\left(k+i_{k} b-\omega\right)_{i} \bar{\partial} x^{i}=(k+v)_{i} \bar{\partial} x^{i} \equiv(k+v) \cdot \bar{\partial} x
\end{gathered}
$$

If we wish to gauge the isometry we introduce gauge fields $A, \bar{A}$, with $\delta_{\epsilon} A=$ $-\partial \epsilon, \delta_{\epsilon} \bar{A}=-\bar{\partial} \epsilon$, and $\delta x^{i}=\epsilon k^{i}(x)$ now with $\epsilon$ a function on the world-sheet. The original action will vary into $\frac{1}{2 \pi} \int\left(J_{k} \bar{\partial} \epsilon+\bar{J}_{k} \partial \epsilon\right)$ which can be cancelled by adding an extra term to the action of the form $\int\left(A \bar{J}_{k}+\bar{A} J_{k}\right) / 2 \pi$. However the new term still varies under the gauge isometry due to the variation of $J_{k}, \bar{J}_{k}$. By adding one new term to the action, $\int k^{2} A \bar{A} / 2 \pi$, the total variation becomes

$$
\delta\left(L+L^{\prime}+L^{\prime \prime}\right)=A \bar{\partial}(\epsilon k \cdot v)-\bar{A} \partial(\epsilon k \cdot v)
$$

where $L$ is given by (2.1) and:

$$
\begin{aligned}
L^{\prime} & =\frac{1}{2 \pi}\left(A \bar{J}_{k}+\bar{A} J_{k}\right) \\
L^{\prime \prime} & =\frac{1}{2 \pi} k^{2} A \bar{A}
\end{aligned}
$$

The anomalous variation (2.8) cannot be cancelled in general unless we include 
extra fields into the action. The simplest choice is a real scalar field $\chi$, contributing to the Lagrangian a term:

$$
L_{\chi}=\frac{1}{2 \pi}(A \bar{\partial} \chi-\bar{A} \partial \chi)
$$

and

$$
\delta_{\epsilon} \chi=-\epsilon k \cdot v
$$

The complete action and transformation rules are:

$$
\begin{gathered}
S_{d+1}=\frac{1}{2 \pi} \int\left[\left(g_{i j}+b_{i j}\right) \partial x^{i} \bar{\partial} x^{j}+\left(J_{k}-\partial \chi\right) \bar{A}+\left(\bar{J}_{k}+\bar{\partial} \chi\right) A+k^{2} A \bar{A}\right] d^{2} \sigma \\
\delta_{\epsilon} x^{i}=\epsilon k^{i}(x) \quad \delta_{\epsilon} \chi=-\epsilon k \cdot v \\
\delta_{\epsilon} A=-\partial \epsilon \quad \delta_{\epsilon} \bar{A}=-\bar{\partial} \epsilon
\end{gathered}
$$

For a genus $g$ world-sheet $\Sigma_{g}$ and compact isometry orbits we may have large gauge transformations. We consider multivalued gauge functions:

$$
\int_{\gamma} d \epsilon=2 \pi n(\gamma) \quad n(\gamma) \in Z
$$

where $\gamma$ is a non-trivial homology cycle in $\Sigma_{g}$. Since we are dealing with abelian isometries it suffices to consider only the toroidal case $g=1$. The variation of $S_{d+1}$ (2.12) is:

$$
\begin{aligned}
\delta S_{d+1} & =\frac{1}{2 \pi} \int(\partial \chi \bar{\partial} \epsilon-\partial \epsilon \bar{\partial} \chi)=\frac{i}{4 \pi} \int_{T} d \chi \wedge d \epsilon \\
& =\frac{i}{4 \pi}\left(\oint_{a} d \chi \oint_{b} d \epsilon-\oint_{a} d \epsilon \oint_{b} d \chi\right)
\end{aligned}
$$

where $a$ and $b$ are the two generators of the homology group of the torus T. Since 
$\epsilon$ is multivalued by $2 \pi Z$, we learn from (2.15) that $\chi$ is multivalued by $4 \pi Z$.

$$
\oint_{\gamma} d \chi=4 \pi m(\gamma) \quad m(\gamma) \in Z
$$

For a non-compact isometry $\delta S_{d+1}=0$ and $d \chi$ may in general have real periods. Thus gauge invariance determines the explicit multivaluedness and the periods of $\chi$.

In duality arguments the first step consists of exhibiting the equivalence of $S_{d+1}$ (2.12) with the original model (2.1) . This in principle follows on spherical topologies by eliminating $\chi$ from $S_{d+1}$. For other topologies we have to be careful with the possible monodromy contributions since $\chi$ is multivalued. If we consider the two terms in $S_{d+1}$ containing $\chi$ and $A, \bar{A}$, we can decompose $d \chi$ into an exact part $d \chi_{0}$ ( $\chi_{0}$ a single valued function) plus a harmonic piece $d \chi_{h}$ which has nontrivial periods. The term $d \chi_{0} \wedge A$ can be integrated by parts, and upon eliminating $\chi_{0}$ it imposes the constraint that $A$ should be flat. However, although $A$ may be flat, it can have non-trivial holonomy about the $a, b$ cycles. The harmonic part of $\chi$ then enforces the triviality of the holonomy of $A$, and this then implies that A is pure gauge and that indeed (2.12) is equivalent to (2.1) . The second step, which produces the dual action, implies the elimination of the gauge field $A$. This is possible because $A$ appears only quadratically in the action. However, since in principle the Killing vector could have fixed points (points where $k^{2}=0$ ), it is perhaps better to think of $S_{d+1}$ (2.12) as the dual action to (2.1).

By construction $S_{d+1}$ is manifestly general covariant, and therefore we have a clear idea of the $d$-dimensional geometrical interpretation of the model. We are working on the quotient of $M$ by the orbits of the isometry group times the $\chi$-space. For a compact isometry $\chi$ lives on a circle and therefore generically one would expect to end up in the product manifold (or orbifold) $\tilde{M}=$ " $\left(M / S^{1}\right) \times S_{\chi}^{1}$ ". Generically we expect topology change as a consequence of duality. However the more delicate issue is whether the dual manifold $\tilde{M}$ is indeed a product or a twisted 
product (non- trivial bundle, etc.). It is also useful to notice that in the previous arguments the structure of $\pi_{1}(M)$ played no rôle. In toroidal compactifications $\pi_{1}\left(T^{d}\right)=Z^{d}$, and together with ordinary momentum states we have winding states describing the way the string wraps around in the compactified dimensions. Duality is described as the symmetry exchanging momentum and winding states. For other manifolds, in particular those where $\pi_{1}(M)=0$ and yet $\tilde{M}$ is not diffeomorphic to $M$, we have to reconsider the mapping between the operators of the two theories.

If we are interested in the explicit form of the dual $\sigma$-model we have to eliminate the gauge field $A$. For Killing vectors with fixed points $\left(k^{2}=0\right)$ this generates singular manifolds. Thus gauge fixing (2.12) in this case produces a singular space whose background interpretation is doubtful whereas (2.12) itself is perfectly welldefined. The simplest way to gauge fix (2.12) is to choose coordinates adapted to the Killing field $k^{i}:\left(\theta, x^{\alpha}\right), k^{i} \partial / \partial x^{i}=\partial / \partial \theta$. This is a representation that depends on the system of coordinate patches used, and the global issues appear as Gribov problems. Using adapted coordinates, and eliminating the gauge field, we obtain after some manipulation the following form for $\tilde{g}, \tilde{b}$ :

$$
\begin{aligned}
& \tilde{g}_{00}=\frac{1}{k^{2}} \\
& \tilde{g}_{0 \alpha}=\frac{v_{\alpha}}{k^{2}} \\
& \tilde{b}_{0 \alpha}=\frac{k_{\alpha}}{k^{2}} \\
& \tilde{g}_{\alpha \beta}=g_{\alpha \beta}-\frac{k_{\alpha} k_{\beta}-v_{\alpha} v_{\beta}}{k^{2}} \\
& \tilde{b}_{\alpha \beta}=b_{\alpha \beta}-\frac{k_{\alpha} v_{\beta}-k_{\beta} v_{\alpha}}{k^{2}}
\end{aligned}
$$

In local adapted coordinates we recover Buscher's transformation. As pointed out before, the global structure is captured directly from $S_{d+1}$ without the need to integrate out the gauge field.

A different way to proceed is to cover the target manifold $M$ with coordinate patches, do patchwise duality transformations, and then try to reconstruct $\tilde{M}$ out 
of the "dual" patches. We will use this procedure in a simple example in the next section for illustrative purposes.

\section{SOME SIMPLE EXAMPLES IN NON-CONFORMAL THEORIES}

In the previous section we analyzed the duality transformation with respect to an abelian symmetry in a manifestly covariant framework to understand the global topology of the dual manifold. It is also instructive to understand the same results explicitly in some examples, especially in those with $\pi_{1}(M)=0$. Since we want to maintain the background interpretation for the $\sigma$-model, we look for geometries with fixed-point free isometries and vanishing fundamental group. The simplest example is to take $M=S U(2)=S^{3}$ with the round metric. Locally $S^{3}$ is certainly equivalent to $S^{2} \times S^{1}$, but not globally. The left action of $S U(2)$ on the round metric is free from fixed points. We may represent $S^{3}$ as a submanifold of $C^{2}$, with coordinates $\left(z_{0}, z_{1}\right)$, satisfying $\bar{z}_{0} z_{0}+\bar{z}_{1} z_{1}=1$. It is also useful to represent $S^{3}$ as a Hopf fibering over $S^{2}, p: S^{3} \rightarrow S^{2}$. When $z_{0} \neq 0, p\left(z_{0}, z_{1}\right)=z=z_{1} / z_{0}$, and when $z_{1} \neq 0, p\left(z_{0}, z_{1}\right)=1 / z=z_{0} / z_{1}$. If $H_{ \pm}$represent the two hemispheres of the two-sphere, the local equivalence of $S^{2} \times S^{1}$ with $S^{3}$ is given by

$$
\begin{gathered}
H_{+}:\left(z, u_{+}\right) \in S^{2} \times S^{1} \rightarrow\left(\frac{u_{+}}{\sqrt{1+|z|^{2}}}, \frac{z u_{+}}{\sqrt{1+|z|^{2}}}\right) \\
H_{-}:\left(1 / z, u_{-}\right) \in S^{2} \times S^{1} \rightarrow\left(\frac{|z| u_{-}}{\sqrt{1+|z|^{2}}}, \frac{|z| u_{-} / z}{\sqrt{1+|z|^{2}}}\right) .
\end{gathered}
$$

The transition function in the equator of the two-sphere is

$$
u_{+}=\frac{|z|}{z} u_{-}=e^{i \phi} u_{-}
$$

which defines the necessary twist in the fiber in order to obtain the three-sphere 
as the global space. We can parametrize the geometry in terms of Euler angles

$$
\begin{aligned}
& z_{0}=e^{i(\chi+\phi) / 2} \cos \theta / 2 \\
& z_{1}=e^{i(\chi-\phi) / 2} \sin \theta / 2
\end{aligned},
$$

where $0 \leq \theta<\pi, 0 \leq \phi<2 \pi, 0 \leq \chi<4 \pi$, corresponding to a parametrization of $S U(2)$ as follows

$$
g=e^{i \chi \sigma_{3} / 2} e^{i \theta \sigma_{1} / 2} e^{i \phi \sigma_{3} / 2} .
$$

In terms of these coordinates the left-invariant one-forms $g^{-1} d g=i \sigma_{a} \omega_{a L} / 2$ are given by

$$
\begin{aligned}
& \omega_{1 L}=\sin \theta \sin \phi d \chi+\cos \phi d \theta \\
& \omega_{2 L}=\sin \phi d \theta-\sin \theta \cos \phi d \chi \\
& \omega_{3 L}=d \phi+\cos \theta d \chi,
\end{aligned}
$$

and the round metric is simply

$$
\begin{aligned}
d s^{2} & =\delta_{a b} \omega_{L}^{a} \omega_{L}^{b} \\
& =d \theta^{2}+d \phi^{2}+2 \cos \theta d \phi d \chi+d \chi^{2} .
\end{aligned}
$$

The isometry we will be interested in corresponds in complex coordinates to the change of the common phase of $z_{0}$ and $z_{1}$. In terms of Euler angles this is $\chi \rightarrow$ $\chi+$ const.. Since the Euler angles are adapted coordinates to this isometry we can apply Buscher's formulae (1.1) to obtain the dual metric

$$
d \tilde{s}^{2}=d \theta^{2}+d \tilde{\chi}^{2}+\sin ^{2} \theta d \phi^{2},
$$

and

$$
\tilde{b}=2 \cos \theta d \tilde{\chi} \wedge d \phi .
$$

this dual metric looks certainly like $S^{2} \times S^{1}$, but at this point it is a bit difficult to discriminate between $S^{2} \times S^{1}$ and $S^{3}$ because Euler angles (like polar coordinates) 
are not globally valid, and we should beware of global topological conclusions obtained in a particular coordinate system. We can be more careful and use a description of $S^{3}$ in terms of two coordinate patches using stereographic projection. In $R^{3}$ we embed $S^{3}$ by the condition $\left(\xi^{0}\right)^{2}+\left(\xi^{i}\right)^{2}=1, i=1,2,3$. The stereographic projection is explicitly given by

$$
x_{i}^{ \pm}=\frac{\xi_{i}}{1 \mp \xi_{0}},
$$

with transition functions

$$
x_{i}^{+}=x_{i}^{-} / x_{-}^{2} \quad x_{-}^{2} x_{+}^{2}=1
$$

The induced metric on $S^{3}$ is the round metric. The Killing vector $k=\partial / \partial \chi$ in the two hemispheres takes the form:

$$
k_{ \pm}^{i}=\left( \pm x_{1} x_{3}-x_{2}, x_{1} \pm x_{2} x_{3}, \pm\left(1+x_{3}^{2}-x_{1}^{2}-x_{2}^{2}\right) / 2\right), \quad k_{ \pm}^{2}=1 .
$$

Applying Buscher's formulae in both patches we obtain the dual metrics:

$$
d \tilde{s}_{ \pm}^{2}=d \tilde{\chi}_{ \pm}^{2}+\left(g_{i j}-k_{i}^{ \pm} k_{j}^{ \pm}\right) d x_{ \pm}^{i} d x_{ \pm}^{j}
$$

$\tilde{\chi}_{ \pm}$are the Lagrange multipliers in each patch. We also generate $b$-fields

$$
\tilde{b}_{ \pm}=-k^{ \pm} \wedge d \tilde{\chi}_{ \pm} \quad k^{ \pm}=k_{i}^{ \pm} d x^{i}
$$

We can patch now $d \tilde{s}_{ \pm}^{2}$ by imposing $\tilde{\chi}_{+}=\tilde{\chi}_{-}$. We also know that there is a redundant degree of freedom in the metric because of gauge invariance. Thus, in order to obtain a metric depending on three coordinates we have to make a gauge choice, for example by choosing $x^{1}=0$. However this is possible as long as $k^{1}(x) \neq 0$. Hence to fix the gauge we have to first look at the fixed surfaces of the three components of the Killing vector. The set of zeroes of $k_{ \pm}^{1}$ is the 
surface $Z_{1}^{ \pm}=\left\{x_{2}= \pm x_{1} x_{3}\right\}$. Similarly $Z_{2}^{ \pm}=\left\{x_{1}=\mp x_{2} x_{3}\right\}$, and $Z_{3}^{ \pm}=\left\{x_{3}^{2}=\right.$ $\left.x_{2}^{2}+x_{1}^{2}-1\right\}$. These sets have vanishing intersections because the Killing vector has no fixed points. In fixing the gauge we can choose $x_{1}=0$ only in $H_{+}-Z_{1}^{+}$. In $Z_{1}^{+}-\left(Z_{1}^{+} \cap Z_{2}^{+}\right)$we can choose $x_{2}=0$, and finally in the intersection between $Z_{1}^{+}$ and $Z_{2}^{+}$we may choose $x_{3}=0$. In this form the gauge is properly fixed and we indeed obtain $S^{2} \times S^{1}$. Incidentally, the correct procedure to fix the gauge solves a small conceptual puzzle. We can cover $S^{3}$ with two simply connected coordinate patches which overlap in a simply connected region. However this is not the case for $S^{2} \times S^{1}$. The extra patches needed to obtain a good cover of this manifold follow from carefully fixing the gauge in order to describe the dual manifold.

The example we have just considered can be extended to an arbitrary compact Lie group $G$. For any abelian subgroup $H$ in $G$ we may consider its left action $h \in H, g \rightarrow h g$. For the principal chiral model,

$$
S=\frac{1}{8 \pi} \int d^{2} \sigma \operatorname{Trg}^{-1} \partial_{\mu} g g^{-1} \partial^{\mu} g .
$$

Once again we can follow the procedure of gauging the subgroup $H$, introducing the Lagrange multiplier etc. to obtain the dual model. We will spare the reader the details, and simply mention the result. After appropriate gauge fixing in this case (labelling correctly the $H$-orbits in $G$ ) we obtain as expected the dual manifold

$$
\tilde{G}=(G / H) \times\{\text { Lagrange multiplier manifold }\} .
$$

We should also be careful about the behavior of the measure in the path integral under this decomposition. However the measure of the original path integral is the Haar measure. It is known [14] that when $\left|\operatorname{det} A d_{G}(h)\right|=\left|\operatorname{det} A d_{H}(h)\right|$ for all $h \in H^{\star}$ there exists a measure on the quotient $G / H$ such that

$$
\int_{G} f(g) d g=\int_{G / H} d g_{H} \int_{H} d h f\left(h g_{H}\right) .
$$

\footnotetext{
$\star A d_{G}(h)$ and $A d_{H}(h)$ are respectively the adjoint actions of $h \in H$ considered as an element of $G$ and $H$.
} 
In the example at the beginning of the section $H=U(1), G=S U(2)$, and the above condition is satisfied. This completes the (semiclassical) proof of the topology change from $S^{3}$ to $S^{2} \times S^{1}$. We will later analyze the mapping between operators of the two models, however we would like to make a few remarks about the mapping between states. In the three-sphere, a natural basis to expand the states is in terms of the Wigner functions (the natural basis of functions on $S U(2)$ ). These functions are labelled by three parameters $D_{m, m^{\prime}}^{j}$, and we can label the vertex operators in terms of them. When we consider the dual space $S^{2} \times S^{1}$, the states on $S^{2}$ are naturally labelled in terms of spherical harmonics $Y_{m}^{l}$. Naively, the states in $S^{1}$ would be labelled by two quantum numbers $\left(n, n^{\prime}\right)$, (momentum and winding respectively) and therefore it would seem that there is a redundancy of states with respect to the spectrum on the three sphere we started with. The resolution of this puzzle is found on the fact that the equation of motion of the Lagrange multiplier field $\chi$ is not given in terms of the free Laplace equation on the world-sheet, but it contains a contribution due to the induced WZW term, which is the analogue of the Dirac monopole connection. In solving this equation, the number of modes with finite action will be cut in half, and therefore the count of states in the original model and its dual agrees.

\section{ABELIAN DUALITY IN WZW MODELS}

A class of Conformal Field Theories (CFT) where the global properties of duality can be controlled in detail are the Wess-Zumino-Witten (WZW) models [15]. On a genus $g$ Riemann surface the action for a simply connected group is

$$
S_{0}[g]=-\frac{k}{2 \pi} \int_{\Sigma_{g}} \operatorname{Tr}\left(g^{-1} \partial g g^{-1} \bar{\partial} g\right)+\frac{i k}{12 \pi} \int_{\Sigma_{g}^{0}} \operatorname{Tr}\left(g^{-1} \partial g\right)^{3}
$$

where $\Sigma_{g}^{0}$ is a filled surface whose boundary is the Riemann surface $\Sigma_{g}$. In these models the duality manipulations can be carried out without reference to any 
coordinate system. This is due to the Polyakov-Wiegmann property (PW) [16]

$$
S_{0}\left[g_{1} g_{2}\right]=S_{0}\left[g_{1}\right]+S_{0}\left[g_{2}\right]-\frac{k}{\pi} \int_{\Sigma_{g}} \operatorname{Tr}\left(g_{1}^{-1} \partial g_{1} \bar{\partial} g_{2} g_{2}^{-1}\right) .
$$

For genus higher than one and for twisted fields this formula receives corrections, however for abelian twists (4.2) is correct in any genus. We consider the following left and right action of an abelian subgroup generated by $H$ :

$$
h_{L}=e^{\cos \alpha \theta H} \quad h_{R}=e^{\sin \alpha \theta H} .
$$

Using PW

$$
S_{0}\left[h_{L} g h_{R}^{-1}\right]=S_{0}[g]+\frac{k}{2 \pi} \int \eta_{\alpha} \partial \theta \bar{\partial} \theta+\frac{k}{2 \pi} \int\left(J_{\alpha} \bar{\partial} \theta-\bar{J}_{\alpha} \partial \theta\right),
$$

where

$$
\begin{aligned}
\eta_{\alpha} & =\sin 2 \alpha \operatorname{Tr} H g H g^{-1}-\operatorname{Tr} H^{2} \\
J_{\alpha} & =2 \sin \alpha \operatorname{Tr} H g^{-1} \partial g \\
\bar{J}_{\alpha} & =2 \cos \alpha \operatorname{Tr} H \bar{\partial} g g^{-1} .
\end{aligned}
$$

This formula represents the effect of a gauge transformation on $S_{0}$ with respect to an arbitrary mixing of left/right abelian actions for some abelian subgroup $H_{\alpha}$. Special cases are $\alpha=0, \pi / 2$ corresponding to pure left- or right-chiral rotations and $\alpha= \pm \pi / 4$, which represent vector and axial transformations. For non-compact $H_{\alpha}$ we take $H_{\alpha}^{\dagger}=H_{\alpha}, H_{\alpha}^{2}=1$ while for compact subgroups we take $H_{\alpha}^{\dagger}=-H_{\alpha}, H_{\alpha}^{2}=$ -1 . In this case we restrict to $\tan \alpha=n_{R} / n_{L}$, a rational number, to avoid ergodic actions. Gauging the WZW Lagrangian (4.1) with respect to the action (4.3) we obtain the dual model

$$
S^{(\alpha)}=S_{0}(g)-\frac{k}{2 \pi} \int A\left(\bar{J}_{\alpha}+t_{2} \bar{\partial} \chi\right)+\frac{k}{2 \pi} \int \bar{A}\left(J_{\alpha}+t_{2} \partial \chi\right)+\frac{k}{2 \pi} \int \eta_{\alpha} A \bar{A},
$$


where $t_{2}=\operatorname{Tr} H^{2}$, with the gauge invariance

$$
\begin{aligned}
& g \rightarrow e^{\cos \alpha \epsilon H} g e^{-\sin \alpha \epsilon H} \\
& A \rightarrow A-d \epsilon \\
& \chi \rightarrow \chi-\epsilon \cos 2 \alpha .
\end{aligned}
$$

In the compact case large gauge transformations correspond to

$$
\oint_{\gamma} d \epsilon=2 \pi r_{\alpha} n(\gamma) \quad r_{\alpha}=\sqrt{n_{L}^{2}+n_{R}^{2}},
$$

where $\gamma$ is a non-trivial homology cycle and $n(\gamma)$ is an integer.

If we integrate the Lagrange multiplier we project into $A=d a$, with $\oint_{\gamma} d a=$ $2 \pi r_{\alpha} n(\gamma)$, and using the PW property together with the invariance of the Haar measure we easily establish the equivalence between (4.1) and (4.5). The explicit form of the dual model follows from $A$-integration, and yields

$$
\tilde{S}=S_{0}\left(g_{c}\right)+\frac{k}{2 \pi} \int \frac{\left(J_{c}+t_{2} \partial \chi\right)\left(\bar{J}_{c}+t_{2} \bar{\partial} \chi\right)}{\eta_{\alpha}},
$$

where $J_{c}=J_{\alpha}\left(g_{c}\right), g_{c} \in G / H_{\alpha}$. The multivaluedness of $\chi$ is as follows

$$
\oint_{\gamma} d \chi=\frac{4 \pi}{r_{\alpha} k t_{2}} n(\chi)
$$

in the case with $H_{\alpha}$ compact, $(n(\chi)$ is an integer $)$ and

$$
\oint_{\gamma} d \chi=l(\chi) \in R \text {. }
$$

Thus we are clearly working in the spaces $G / H_{\alpha} \times S_{\chi}^{1}$ for compact $H_{\alpha}$ and $G / H_{\alpha} \times$ $\tilde{R}_{\chi}$ for non-compact $H_{\alpha}$ for generic $\alpha$. By looking at the term in the action quadratic in the $\chi$-field we can determine the radius of the circle $S_{\chi}^{1}$ to be $R_{\chi}=$ $2 / r_{\alpha} \sqrt{k \eta_{\alpha}}$. 
We can easily check involution using again the PW property. The dual model has an obvious isometry with respect to $\chi$-shifts, and by applying duality with respect to this symmetry together with the PW property, we arrive after a trivial rescaling of variables to the original theory.

A particularly interesting case corresponds to the chiral theories $\alpha=0, \pi / 2$. These theories are self-dual after the first duality transformation. For example, for $\alpha=0, J_{c}=0$ and $\eta_{\alpha}=-t_{2}$ :

$$
\tilde{S}_{\alpha=0}=S_{0}\left(g_{c}\right)-\frac{k}{2 \pi} \int \bar{J}_{c} \partial \chi-\frac{k}{2 \pi} \int t_{2} \partial \chi \bar{\partial} \chi
$$

and using the PW property once more we arrive at the original model. Thus the WZW-model is self-dual with respect to pure left- or right-abelian duality.

As a particularly nice example of the previous considerations we have the duality between 3d-rotating black holes and 3d-charged black strings [17]. The 3d-black hole is given by the gauged WZW-model

$$
S \widetilde{L(2, R)} / S O(1,1)_{\alpha}^{Z}
$$

where the notation means that we have to identify points with integer spacing along the $S O(1,1)_{\alpha}$-orbits. $\alpha$ is related here to the black hole angular momentum, and we effectively deal with a compact isometry. $S \widetilde{L(2, R)}$ is the universal covering of $S L(2, R)$. Under duality,

$$
S \widetilde{L(2, R)} / S O(1,1)_{\alpha}^{Z} \rightarrow \widetilde{S(2, R)} / S O(1,1)_{\alpha} \times S_{\phi}^{1}
$$

The model on the right is the three-dimensional charged black string. For the particular case of $\alpha=-\pi / 4$ we obtain the static three-dimensional black hole and the uncharged string, which becomes (at tree level) a simple product of the two-dimensional black hole and $S^{1}$. 
In conclusion, we have generic topology change induced by duality even at the semiclassical level. Quite generally the topological properties of the dual space depend only on the embedding structure of the isometry orbits, in particular on its compactness or non-compactness. Whether $\pi_{1}(M)$ is trivial seems to be, to a large extent irrelevant.

\section{ORDER-DISORDER MAPPING}

Duality transformations map not only the actions of the $\sigma$-models under consideration but also provide an explicit dictionary between the Hilbert spaces, and the physical operators. This is the order-disorder transformation in the context of Kramers-Wannier duality in Statistical Mechanics, or the winding-Kaluza-Klein mode mapping in toroidal compactifications in string theory. To be more precise, duality in its more general form is an equality of the form:

$$
\left\langle O_{1} \ldots O_{n}\right\rangle=\left\langle\tilde{O}_{1} \ldots \tilde{O}_{n}\right\rangle,
$$

for some dual operators $\tilde{O}_{i}$ to be determined. Here we consider local order operators (in adapted coordinates) of the form

$$
P\left(\partial_{z}, \partial_{\bar{z}}\right) f\left(\theta, x^{\alpha}\right)(z, \bar{z})
$$

for $f$ some function on the target space-time $M$ being the analogue of a tachyon operator. For example, in group manifolds $M=G$, we may diagonalize the action of $H_{\alpha}$ for the basis functions

$$
f_{p_{L}, p_{R}}(g)=f_{p_{L}, p_{R}}\left(e^{\theta_{L} H} g_{c} e^{-\theta_{R} H}\right)=e^{i\left(p_{L} \theta_{L}-p_{R} \theta_{R}\right)} f_{p_{L}, p_{R}}\left(g_{c}\right) .
$$

Since $g_{c}$ is a spectator field in duality transformations, we can consider operators 
of the form

$$
V_{p}=e^{i p \theta}
$$

and in general,

$$
\mathcal{O}=P\left(\partial_{z}, \partial_{\bar{z}}\right) e^{i p \theta}
$$

Thus, in establishing (5.1) it suffices to concentrate on tachyon operators.

For non-compact $H_{\alpha} p \in R$ (or more generally $p \in C$ ) while in the compact case $p \in Z$ (assuming that $\theta$ is identified modulo $2 \pi$ ). It is easy to perform the duality transformation in the functional integral (here we consider only tree-level duality):

$$
\left\langle V_{p_{1}}\left(z_{1}\right) \ldots V_{p_{n}}\left(z_{n}\right)\right\rangle=\int D \theta D x^{\alpha} V_{p_{1}}\left(z_{1}\right) \ldots V_{p_{n}}\left(z_{n}\right) e^{-S\left(\theta, x^{\alpha}\right)} .
$$

First we fix a point on the surface $\Sigma$, say $P=\infty$ and write,

$$
\begin{aligned}
\prod_{i} V_{p_{i}} & =\prod_{j} e^{i p_{j}\left(\theta\left(z_{j}\right)-\theta(\infty)\right)} \prod_{j} e^{i p_{j} \theta(\infty)} \\
& =\prod_{j} e^{i p_{j} \int_{\infty}^{z_{j}} d \theta} e^{i \sum_{j} p_{j} \theta(\infty)}
\end{aligned}
$$

As usual we may fix the translational symmetry zero mode in $\theta$ by the requirement of charge conservation $\sum_{j} p_{j}=0$, and the integrals $\int_{\infty}^{z_{j}} d \theta=\int_{\gamma_{j}} d \theta$ go along arbitrary cuts drawn on $\Sigma$. Now we can use a first order formalism. We can further transform the operators to the form

$$
\int_{\infty}^{z} d \theta=\frac{1}{2 \pi} \oint_{\gamma_{z}} d \theta \alpha=\frac{1}{2 \pi} \int_{\Sigma} d \alpha \wedge d \theta
$$

for $\alpha$ a function with $2 \pi$ jumps on $\gamma_{z}$, ie it is an "angular variable" centered at $z$. Now we may gauge the model to define the following correlator on the $d+1$ - 
dimensional theory:

$$
\begin{gathered}
\left\langle\prod_{j} V_{p_{j}}\right\rangle=\int \frac{D \theta D x^{\alpha} D A D \chi}{\left|H_{\alpha}\right|} e^{-S_{e f f}\left(p_{j}, A, \theta, x^{\alpha}, \chi\right)} \\
S_{e f f}=S_{d+1}\left(\theta, x_{\alpha}, A, \chi\right)+\frac{i}{2 \pi} \sum_{j} p_{j} \int(d \theta+A) \wedge d \alpha_{j} .
\end{gathered}
$$

Where $\left|H_{\alpha}\right|$ is the volume of the gauge group generated by the isometry. Thus the dual operators in the $d+1$-dimensional sense are given by the non-local expressions:

$$
W_{p}^{(d+1)}(z)=e^{i p \int_{\infty}^{z}(d \theta+A)}=e^{i p \int_{\gamma z} D \theta}
$$

We can also work out the $d$-dimensional gauge invariant form by integrating out A. Upon gauge fixing,

$$
S_{e f f} \rightarrow S_{d+1}\left(\theta=0, x_{\alpha}, A, \phi\right)+\frac{1}{\pi} \sum_{j} p_{j} \int\left(A \bar{\partial} \alpha_{j}-\bar{A} \partial \alpha_{j}\right)
$$

and after integrating over $A$ we obtain a frustrated partition function

$$
\left\langle\prod_{j} V_{p_{j}}\right\rangle=\int D x^{\alpha} D \phi e^{-\tilde{S}_{d}\left(\phi^{*}, x_{\alpha}\right)}
$$

where $\phi^{*}$ is the frustrated field

$$
d \phi^{*}=d \phi+\sum_{j} 2 p_{j} d \alpha_{j}, \quad \oint_{\left|z-z_{j}\right|=\epsilon} d \phi^{*}=4 \pi p_{j}
$$

Hence the dual operators to the $V_{p}$ 's are genuine winding operators for compact $H_{\alpha}$ with charge $2 p$. For non-compact $H_{\alpha}$ we have vortex lines with real charge $2 p$. This is expected from the study of the higher genus partition functions. 
Recalling the explicit form of the $\phi$-sector in the dual model,

$$
\begin{gathered}
\tilde{S}_{\phi} \sim \frac{1}{2 \pi} \int \frac{1}{\xi^{2}} \partial \phi \bar{\partial} \phi-\frac{i}{4 \pi} \int \frac{1}{\xi^{2}} j \wedge d \phi \\
j=\left(J_{c}, \bar{J}_{c}\right)
\end{gathered}
$$

we find it is quadratic in $\phi$, so that we can still sharpen the form of the dual operators. It proves convenient to choose $\alpha_{j}$ as solutions to the pull-back Laplace equation:

$$
d\left(\xi^{-2} * d \alpha_{j}\right)=0
$$

Then expanding the frustrated action

$$
\begin{aligned}
\tilde{S}_{\phi^{*}} & =\tilde{S}_{\phi}+\tilde{S}_{\sum_{j} 2 p_{j} \alpha_{j}} \\
& =\tilde{S}_{\phi}+\sum_{i j} p_{i} p_{j} \int_{\gamma_{i}} \xi^{-2} * d \alpha_{j}-\sum_{j} \frac{i p_{j}}{2 \pi} \int d \alpha_{j} \wedge j \xi^{-2} .
\end{aligned}
$$

Since $\tilde{S}_{\phi}$ is at most quadratic, and the $\alpha_{j}$ 's are solutions to the Laplace equation, we can write

$$
\left\langle\prod_{j} V_{p_{j}}\right\rangle_{S}=\left\langle\prod_{j} W_{p_{j}}\right\rangle_{\tilde{S}}
$$

for

$$
W_{p}=e^{p \int_{\infty}^{z} \xi^{-2} * d \phi}
$$

Note that for constant $\xi^{2}, \phi$ admits a holomorphic decomposition $\phi(z, \bar{z})=\phi(z)+$ $\bar{\phi}(\bar{z})$, and we may integrate the operator to

$$
W_{p}=e^{-\frac{i p}{\xi^{2}}(\phi(z)-\bar{\phi}(\bar{z}))}
$$

the usual flat winding mode. For non-constant $\xi^{2}$ this is not possible in general, 
the operator remains string-like and the dual operators take the form

$$
P\left(\frac{1}{i p} \partial_{z}, \frac{1}{i p} \partial_{\bar{z}}\right) e^{-i p \int^{z}\left(d z \partial_{z} \phi-d \bar{z} \partial_{\bar{z}} \phi\right) / \xi^{2}} .
$$

For example, the dual of $\partial \theta \bar{\partial} \theta e^{i p \theta}$ is given by

$$
\frac{1}{i p} \partial_{z} \partial_{\bar{z}} \frac{\phi}{\xi^{2}} e^{-i p \int^{z}\left(d z \partial_{z} \phi-d \bar{z} \partial_{\bar{z}} \phi\right) / \xi^{2}}
$$

Two conclusions can be drawn from this analysis:

1). The semiclassical algorithm allows for an explicit parametrization of dual operators also for curved backgrounds, where an exact Conformal Field Theory construction is not known. We find in general non-local operators which cannot be made local by holomorphic factorization.

2). The physics of the dual model depends on the explicit operator mapping. There is always a translation dictionary involved. For example, for a non-compact isometry group we have to quantize the dual model as a vortex gas rather than counting continuous embeddings in the target space.

\section{REMARKS ON DUALITY AND THE COSMOLOGICAL CONSTANT}

A striking feature of duality is the fact that the cosmological constant, defined as the asymptotic value of the curvature scalar is not in general invariant under duality. One is led to wonder to what extent the cosmological constant is a string observable. This fact noticed in [17] implies that the usual definition of $\Lambda$ from the low-energy effective action is not satisfactory. Even at large distances, if duality is not broken there is a symmetry between local (momentum) modes and non-local (winding) modes. The contribution to the cosmological constant of the massless sector might be cancelled by the tower of massive states always present in String Theory (proposals along these lines using the Atkin-Lehner symmetry were advanced by G. Moore [18]). 
We study now the behavior of the scalar curvature under duality. If the spacetime metric in the $\sigma$-model takes the form

$$
d s^{2}=g_{i j} d x^{i} d x^{j} \quad i, j=0,1,2, \ldots, d-1,
$$

where $x^{0}$ is adapted to the isometry $k=\partial / \partial x^{0},(6.1)$ can be written as

$$
\begin{aligned}
d s^{2} & =\left(e^{0}\right)^{2}+\left(g_{\alpha \beta}-\frac{k_{\alpha} k_{\beta}}{k^{2}}\right) d x^{\alpha} d x^{\beta} \\
e^{0} & =k d x^{0}+\frac{k_{\alpha}}{k} d x^{\alpha} \\
k^{2} & =k_{i} k^{i}=g_{00} \quad k_{\alpha}=g_{0 \alpha} .
\end{aligned}
$$

Buscher's transformation leads to a dual metric

$$
\begin{aligned}
d \tilde{s}^{2} & =\left(\tilde{e}^{0}\right)^{2}+\left(g_{\alpha \beta}-\frac{k_{\alpha} k_{\beta}}{k^{2}}\right) d x^{\alpha} d x^{\beta}, \\
\tilde{e}^{0} & =\frac{1}{k}\left(d \tilde{x}^{0}+v_{\alpha} d x^{\alpha}\right)
\end{aligned}
$$

$\tilde{x}^{0}$ being the Lagrange multiplier and $v$ is defined as in section 2 by $k^{l} H_{l i j}=$ $-\partial_{[i} v_{j]}, H=d b$. The dual scalar curvature following from (6.3) is

$$
\begin{gathered}
\tilde{R}=R-\frac{4}{k^{2}} g^{\alpha \beta} \partial_{\alpha} k \partial_{\beta} k+\frac{4}{k} \Delta_{q}^{d-1} k+ \\
\frac{1}{k^{2}} H_{0 \alpha \beta} H^{0 \alpha \beta}-\frac{k^{2}}{4} F_{\alpha \beta} F^{\alpha \beta},
\end{gathered}
$$

where $\Delta_{q}^{d-1}$ is the $(d-1)$-dimensional Laplacian for the metric $g_{\alpha \beta}^{q}=g_{\alpha \beta}-\frac{k_{\alpha} k_{\beta}}{k^{2}}$, and $F_{\alpha \beta}=\partial_{\alpha} A_{\beta}-\partial_{\beta} A_{\alpha}$ with $A_{\alpha}=k_{\alpha} / k^{2}$. (6.4) can be rewritten as

$$
\tilde{R}=R+4 \Delta \log k+\frac{1}{k^{2}} H_{0 \alpha \beta} H^{0 \alpha \beta}-\frac{k^{2}}{4} F_{\alpha \beta} F^{\alpha \beta} .
$$

From (6.5) we see that the only way to "flatten" negative curvature is by having torsion in the initial space-time. Otherwise the dual of an asymptotically negatively 
curved space time is a space of the same type. Positive curvature seems however easier to flatten in view of (6.5). We can also construct the dual torsion

$$
\begin{aligned}
\tilde{H}_{0 \alpha \beta} & =-\frac{1}{2} F_{\alpha \beta} \\
\tilde{H}_{\alpha \beta \rho} & =H_{\alpha \beta \rho}-\frac{3}{k^{2}} H_{0[\alpha \beta} k_{\rho]}-\frac{3}{2} F_{[\alpha \beta} v_{\rho]} .
\end{aligned}
$$

Since

$$
\sqrt{g}=k^{2} \sqrt{\tilde{g}}
$$

and the modulus of $k$ can be expressed in terms of the dilaton transformation properties,

$$
\tilde{\phi}=\phi-2 \log k
$$

we obtain

$$
\tilde{R}+e^{\phi-\tilde{\phi}} \tilde{H}_{0 \alpha \beta}^{2}+2 \Delta \tilde{\phi}=R+e^{\tilde{\phi}-\phi} H_{0 \alpha \beta}^{2}+2 \Delta \phi
$$

which can be used to show the duality invariance of the string effective action to leading order in $\alpha^{\prime}$, as first noticed in [7].

The change of the cosmological constant under duality is not only peculiar to three-dimensions [17] but rather generic. This raises the physical question of whether in the context of String Theory the value of the cosmological constant can be inferred from the asymptotic (long distance) behavior of the Ricci tensor. If duality is not broken, the answer seems to be in the negative, and it makes the issue of what is the correct meaning of the cosmological constant in String Theory yet more misterious. 


\section{EXAMPLES OF NON-ABELIAN DUALITY}

\subsection{Generalities and $S U(2)$ Duality}

In this section we would like to explore some examples and properties of the non-abelian generalization of duality proposed in [12]. The philosophy in [12] is to generalize the gauging procedure in [11] to manifolds with non-abelian isometry groups. This method leads to an apparent equivalence between two $\sigma$-models with vastly different topologies and geometries. There are some fundamental differences between abelian and non-abelian duality transformations which are worth pointing out.

1. In the context of Statistical Mechanics, duality transformations are applied to models defined on a lattice $L$ with physical variables taking values on some abelian group $G$. The duality transformation takes us from the triplet $(L, G, S[g]$ ), where $S[g]$ is the action depending on some coupling constants labelled collectively by $g$ to a model $\left(L^{*}, G^{*}, S^{*}\left[g^{*}\right]\right)$ on the dual lattice $L^{*}$ with variables taking values on the dual group $G^{*}$ and with some well-defined action $S^{*}\left[g^{*}\right]$. For abelian groups, $G^{*}$ is the representation ring, itself a group, and when we apply the duality transformation once again we obtain the original model. As soon as the group is non-abelian the previous construction breaks down because the representation

ring of $G$ is not a group [1]. In particular the non-abelian duality transformations cannot be performed again to obtain the model we started with.

2. In the continuum, and for abelian duality, the number of abelian symmetries in the original and the dual models is the same. The dual abelian isometries correspond to constant shifts of the associated Lagrange multipliers (the non-abelian part of the original isometry group is probably realized non-locally). In the nonabelian case we systematically lose symmetries. The non-abelian isometry group of the dual space is always smaller, and the original isometry group is not realized locally in the dual theory. It is a difficult problem to find the original theory if we are only given its non-abelian dual. 
For the above two reasons it is somewhat of a misnomer to call non-abelian duality a duality transformation. We will nevertheless follow the nomenclature in the literature. We should also mention that some of the most interesting examples considered below are not conformally invariant field theories, although one could imagine in principle their embedding in conformally invariant $(2,0)$ or $(2,2)$ supersymmetric $\sigma$-models. We have not attempted to carry this out.

Following the outline in [12] we begin with the standard $\sigma$-model on a group manifold $G$.

$$
S(g)=-\frac{k}{2 \pi} \int \operatorname{Tr}\left(g^{-1} \partial g g^{-1} \bar{\partial} g\right)
$$

The action is invariant under the left- and right-action of elements of $G, g \rightarrow$ $h_{1} g h_{2}^{-1} . k$ in (7.1) needs not be an integer since we are not including the WZW term. Since we want to maintain as far as possible the background independence of our manipulations, we perform non-abelian duality with respect to some sugbroup $H$ of $G$ acting without fixed points. For instance we can take a subgroup $H$ acting by left-multiplication. Gauging $H$ and introducing Lagrange multipliers in its adjoint representation, we obtain:

$$
\begin{gathered}
S(g, A, \chi)=-\frac{k}{2 \pi} \int \operatorname{Tr}\left(g^{-1} \partial g g^{-1} \bar{\partial} g+A \bar{\partial} g g^{-1}+\bar{A} \partial g g^{-1}+\right. \\
\bar{A} A+\chi(\partial \bar{A}-\bar{\partial} A+[A, \bar{A}]))
\end{gathered}
$$

(7.2) is invariant under the gauge transformations

$$
g \rightarrow h g \quad A \rightarrow h(A+d) h^{-1} \quad \chi \rightarrow h \chi h^{-1}
$$

To explore non-abelian duality we choose the largest non-abelian subgroup of $G$ acting without fixed points, namely $G$ itself acting on the left. Rather than solving for $A$ in (7.2) and then gauge fixing, we first make the change of variables $A \rightarrow$ $g^{-1}(A+d) g, \chi \rightarrow g^{-1} \chi g$. Then $g$ disappears from the action. This is equivalent 
to choosing the unitary gauge $g=1$. The gauge fixed action becomes

$$
S(A, \chi)=-\frac{k}{2 \pi} \int \operatorname{Tr}(\bar{A} A+\chi F(A)) .
$$

Using the equations of motion for $A$,

$$
\bar{A}+\bar{\partial} \chi+[\bar{A}, \chi]=0
$$

(7.4) becomes

$$
S(A, \chi)=\frac{k}{2 \pi} \int \operatorname{Tr}(\bar{A} \partial \chi)
$$

Solving $\bar{A}$ in terms of the Lagrange multipliers from (7.5) leads to the dual action. Note that for compact groups (7.5) can always be solved without singularities.

The obvious example to consider is $G=S U(2)$. Take as generators $T_{i}=$ $\sigma_{i} / i \sqrt{2} . \bar{A}$ satisfies:

$$
\left(\delta^{i j}+\sqrt{2} \epsilon^{i j k} \chi^{k}\right) \bar{A}^{j}=-\bar{\partial} \chi^{j} .
$$

Solving for $\bar{A}$ and substituting in (7.6) leads to

$$
\tilde{S}(\chi)=\frac{k}{4 \pi} \int\left[\frac{1}{1+\chi^{2}}\left(\delta_{i j}+\chi_{i} \chi_{j}\right)-\frac{1}{1+\chi^{2}} \epsilon_{i j k} \chi_{k}\right] \partial \chi^{i} \bar{\partial} \chi^{j}
$$

In (7.8) $\chi^{2}=\delta_{i j} \chi^{i} \chi^{j}$, and we have absorbed a factor of $\sqrt{2}$ in $\chi$. The dual metric is therefore,

$$
d \tilde{s}^{2}=\frac{1}{1+\chi^{2}}\left(\delta_{i j}+\chi_{i} \chi_{j}\right) d \chi^{i} d \chi^{j}
$$

Using polar coordinates,

$$
d \tilde{s}^{2}=d r^{2}+\frac{r^{2}}{1+r^{2}}\left(d \theta^{2}+\sin ^{2} \theta d \phi^{2}\right) .
$$

Asymptotically as $r \rightarrow \infty$ this is the standard metric on $R \times S^{2}$, and for $r \rightarrow 0$ 
the space looks like $R^{3}$. The scalar curvature for (7.10)

$$
R=2 \frac{\left(9+3 r^{2}+r^{4}\right)}{\left(1+r^{2}\right)^{2}}
$$

is free of singularities as expected because the group acts without fixed points. Hence, at tree level, the non-abelian dual of $G=S U(2)$ with respect to its leftaction is the Lie algebra of $S U(2)$ but with a metric reminiscent of Witten's $S L(2, R) / U(1)$ Euclidean black hole metric [19]. According to [12] the dilaton changes into

$$
\tilde{\phi}=\phi-\log \left(1+\chi^{2}\right)
$$

Notice that $\tilde{S}(\chi)$ contains less symmetry than the original model. (7.1) is invariant under $g \rightarrow h_{1} g h_{2}^{-1}$. Since the dual variables are the gauge invariant combinations $g^{-1} \chi g$, we are left with the action of $S U(2)$ on the right. We can proceed to perform the duality transformation with respect to this residual group. Now $\chi=0$ is a fixed point of the isometry group and the resulting space will be singular. We gauge the symmetry by replacing derivatives by covariant derivatives $D \chi=\partial \chi+[A, \chi]$, and then we add the new Lagrange multiplier $\lambda$ to obtain

$\tilde{S}(\chi, A, \lambda)=\frac{k}{4 \pi} \int\left(-\frac{\operatorname{Tr}(D \chi \bar{D} \chi)}{1+\rho^{2}}-\frac{1}{1+\rho^{2}} \epsilon_{i j k} \chi_{k} D \chi_{i} \bar{D} \chi_{j}+\frac{\partial \rho \bar{\partial} \rho}{1+\rho^{2}}+\operatorname{Tr} \lambda F(A)\right)$

with

$$
\rho=\sqrt{\chi_{i} \chi_{i}}
$$

Before integrating the gauge field, we fix the non-abelian part of the gauge by choosing the unitary-like gauge

$$
\chi=\frac{1}{i \sqrt{2}}\left(\begin{array}{cc}
\rho & 0 \\
0 & -\rho
\end{array}\right)
$$


We fix the residual $U(1)$ gauge invariance as follows: Since $\lambda=\lambda_{a} \sigma^{a} / i \sqrt{2}$,

$$
\lambda=\frac{1}{i \sqrt{2}}\left(\begin{array}{cc}
\lambda_{3} & \lambda_{1}-i \lambda_{2} \\
\lambda_{1}+i \lambda_{2} & -\lambda_{3}
\end{array}\right)
$$

write

$$
\lambda_{1}=r \cos \theta \quad \lambda_{2}=r \sin \theta
$$

and choose the gauge

$$
\theta=0
$$

This fixes the gauge completely and the new dual variables are $\left(\rho, r, \lambda_{3}\right)$. Eliminating $A$ and after some manipulations we obtain

$$
\begin{gathered}
\tilde{\tilde{S}}=\frac{k}{4 \pi} \int\left(\partial \rho \bar{\partial} \rho+\frac{1+\rho^{2}}{2 \rho^{2}} \partial r \bar{\partial} r+\left(\frac{1+\rho^{2}}{2 r \rho^{2}} \lambda_{3}+\frac{\rho}{\sqrt{2} r}\right)\right. \\
\left.\left(\partial r \bar{\partial} \lambda_{3}+\partial \lambda_{3} \bar{\partial} r\right)+\left(\frac{1+\rho^{2}}{2 r^{2} \rho^{2}} \lambda_{3}^{2}+\frac{\rho^{2}}{r^{2}}+\frac{\sqrt{2} \rho}{r^{2}} \lambda_{3}\right) \partial \lambda_{3} \bar{\partial} \lambda_{3}\right) .
\end{gathered}
$$

The new dilaton is

$$
\tilde{\tilde{\phi}}=\phi-\log \frac{\rho^{2} r^{2}}{2}
$$

The double dual has curvature singularities at $\rho=0$ and $r=0$. The Lagrangian (7.17) has no trace of any of the original $S U(2)_{L} \times S U(2)_{R}$ symmetries or of the $S U(2)$ symmetry of the dual Lagrangian. There are no symmetries left at all. It is quite unclear also how to go back from the dual to the original action in contrast with the abelian case. 


\subsection{The Dual of $S L(2, R)_{L}$}

A more interesting example from the space-time point of view is as above but with $S U(2)$ replaced by $S L(2, R)$. The result is simply obtained by analytically continuing the $S U(2)$ formulas according to the rules $\chi_{1} \rightarrow i \chi_{1}, \chi_{2} \rightarrow \chi_{2}, \chi_{3} \rightarrow$ $i \chi_{3}$ (plus a change $k \rightarrow-k$ to have signature $(-,+,+)$ ). We obtain the dual Lagrangian

$$
\begin{gathered}
\tilde{S}(\chi)=\frac{k}{4 \pi} \int\left[\frac{1}{1-\chi^{2}}\left(\eta_{i j}-\chi_{i} \chi_{j}\right)-\frac{1}{1-\chi^{2}} \epsilon_{i j k} \chi_{k}\right] \partial \chi^{i} \bar{\partial} \chi^{j}, \\
\chi^{2}=\eta^{i j} \chi_{i} \chi_{j} \quad \eta=\operatorname{diag}(1,-1,1) .
\end{gathered}
$$

Using the analogue of polar coordinates we can distinguish two regions: $\chi^{2}>0$ and $\chi^{2}<0$. The dual metric takes the form

$$
\begin{aligned}
\chi^{2}>0, \quad d \tilde{s}_{I}^{2} & =\frac{1}{4 \rho} d \rho^{2}-\frac{\rho}{1-\rho} d \eta^{2}+\frac{\rho}{1-\rho} \cosh ^{2} \eta d \phi^{2} \\
\rho & =\chi^{2} ; \quad \eta \in R ; \quad \phi \in[0,2 \pi] \\
\chi^{2}<0 \quad d \tilde{s}_{I I}^{2} & =-\frac{1}{4 \rho} d \rho^{2}+\frac{\rho}{1+\rho} d \eta^{2}+\frac{\rho}{1+\rho} \sinh ^{2} \eta d \phi^{2} \\
\rho & =-\chi^{2},
\end{aligned}
$$

with in region I

$$
\begin{aligned}
& \chi_{1}=\rho^{1 / 2} \cos \phi \cosh \eta \\
& \chi_{3}=\rho^{1 / 2} \sin \phi \cosh \eta \\
& \chi_{2}=\rho^{1 / 2} \sinh \eta,
\end{aligned}
$$

and similarly in region II with minor changes. The scalar curvatures are

$$
R_{I}=-2 \frac{\left(\rho^{2}-3 \rho+9\right)}{(\rho-1)^{2}} \quad R_{I I}=-2 \frac{\left(\rho^{2}+3 \rho+9\right)}{(\rho+1)^{2}} .
$$

$R_{I}$ has a singularity at $\rho=1$. This is not due to fixed points but rather to the non-compactness of the group (infrared problem). The dual space has interesting 
global properties. It is an anti-DeSitter (AdS) space-time. $\rho=0$ is a coordinate singularity where the metric goes from $(-,+,+)$ in region II to $(+,-,+)$ in region I, and $(+,+,-)$ beyond the singularity. Thus $\rho=0$ is likely to be a horizon. To study the causal structure we can ignore the $\phi$-dependence. Each point $(\rho, \eta)$ is a circle of radius $(\rho /(1-\rho))^{1 / 2} \cosh \eta$ in region I and of radius $(\rho /(1+\rho))^{1 / 2}|\sinh \eta|$ in region II. Making $\rho \rightarrow-\rho$ in region II we cover the space-time with

$$
d \tilde{s}^{2}=-\frac{\rho}{1-\rho} d \eta^{2}+\frac{1}{4 \rho^{2}} d \rho^{2}
$$

The $\rho=0$ coordinate singularity can be eliminated by going to "tortoise" coordinates

$$
\rho^{*}=\frac{1}{2} \log \left|\frac{1-\sqrt{1-\rho}}{1+\sqrt{1-\rho}}\right|+\sqrt{1-\rho} ; \quad \rho<1,
$$

so that

$$
d \tilde{s}^{2}=-\frac{\rho}{1-\rho} d \eta^{2}+\frac{\rho}{1-\rho} d \rho^{* 2} .
$$

Region II in these coordinates is covered by $-\infty<\rho^{*}<\infty$. Going to null coordinates $u=\rho^{*}-\eta, v=\rho^{*}+\eta$, and using Kruskal-like coordinates,

$$
\bar{u}=e^{u} \quad \bar{v}=e^{v},
$$

region II is covered with $0<\bar{u}<\infty, 0<\bar{v}<\infty$. In terms of these coordinates we can extend the manifold to a larger manifold $\tilde{M}$

$$
d \tilde{s}^{2}=\frac{\rho}{1-\rho} e^{-2 \rho^{*}} d \bar{u} d \bar{v} \quad-\infty<\bar{u}, \bar{v}<\infty .
$$

The extended region $-\infty<\bar{u}, \bar{v}<0$ is isomorphic to region I and the transition takes place analytically. In Kruskal coordinates the horizon is at $\bar{u}=0, \bar{v}=0$. The Penrose diagram for $\tilde{M}$ appears in fig. 1 . 


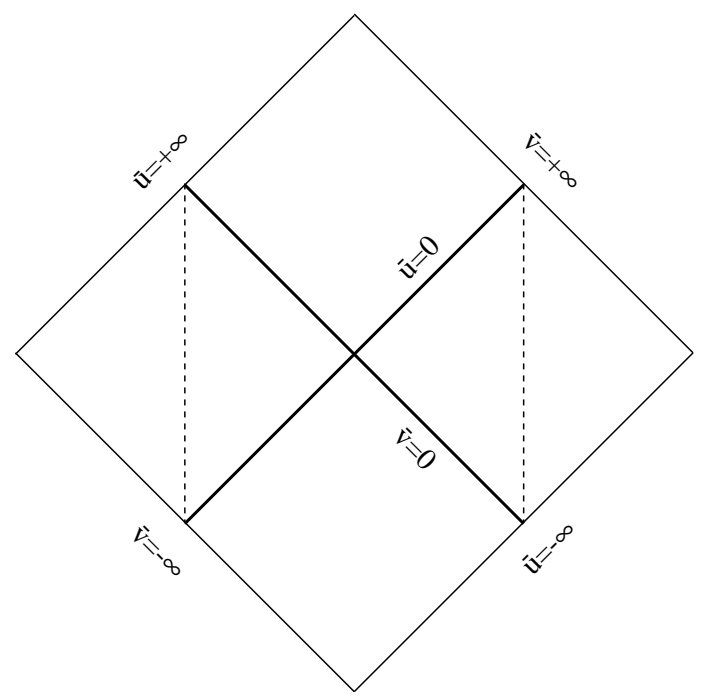

Fig.1: Penrose diagram for $\tilde{M}$.

The Penrose diagram describes a black-hole space-time with a time-like singularity. Asymptotically $\tilde{M}$ has negative scalar curvature with metric $d s^{2}=$ $-d \rho^{2}+d \eta^{2}+\sinh ^{2} \eta d \phi^{2}$. We have thus found a black-hole space-time by dualizing AdS space with respect to the non-abelian symmetry group $S L(2, R)_{L}$. This space-time is different from the three-dimensional space-time found in [20] which arises from identifying points in AdS space by a discrete subgroup of $S O(2,2)$. A time-like singularity appears there, but it is not a curvature singularity. It is a causal singularity beyond which closed time-like curves appear.

In the dual model we still have an $S L(2, R)$-isometry group, and we can again dualize with respect to it. The formulae are similar to those obtained for the $S U(2)$ case after analytic continuation. We will not spell out the details here.

An important remark is that even though the space-time obtained is potentially interesting in the subject of string black holes, we have obtained the result starting with a theory which is not conformally invariant. It is likely that conformal invariance can be gained by embedding the model in a $(2,0)$ supersymmetric theory, but we have not yet explored this possibility. 
An example where one can start directly with a conformally invariant field theory is the $S L(2, R)$-WZW theory if we perform duality with respect to the vector action of $S L(2, R)$. This model leads to a rather singular space-time and presumably the correct way to think about the dual theory is without the elimination of the gauge field and using BRST techniques. In this model the gauge fixing is rather delicate because we have to distinguish the elliptic, parabolic and hyperbolic regions of $S L(2, R)$. We have not found a nice physical interpretation of the dual space-time as a black-hole or any other simple space-time, and will just present an outline of the results in the appendix.

\subsection{Non-Abelian Frustrations}

To conclude this chapter we would like to address some of the problems in the determination of the global properties of non-abelian dual spaces and the current difficulties one finds in trying to obtain the operator mapping. In particular it should also be interesting to determine the form of this transfomation for higher genus Riemann surfaces. Given that duality transformations are versions of Kramers-Wannier duality, it seems at first sight paradoxical that no use of nonabelian duality has been made in Statistical Mechanics. The reason is simple as mentioned at the beginning of the chapter. For non-abelian groups the set of representations does not form a group. To be more precise, duality always proceeds by first transforming to a first order formalism (using gauge fields) with constraints. When the constraints are solved we get back the original model. In the abelian case the constraints take the form

$$
\prod_{z} \delta(d A) \prod_{i} \delta\left(\oint_{a_{i}} A\right) \delta\left(\oint_{b_{i}} A\right)
$$

where $a, b$ are the non-trivial homology cycles of a genus-g Riemann surface. In the non-abelian case similar gauge invariant constraints are expressed in non-local 
form in terms of Wilson loops (we think of hte theory as defined on a lattice)

$$
\prod_{\text {plaquettes }} \delta\left(W_{\text {plaquettes }}\right) \prod_{i} \delta\left(W_{a_{i}}\right) \delta\left(W_{b_{i}}\right),
$$

where the $a, b$ 's are now understood as generators of the homotopy of the surface, and

$$
W_{\gamma}=P e^{\int_{\gamma} A} .
$$

Delta-functions in (7.28) can be expanded in Fourier modes and we obtain local terms in the action for the Lagrange multipliers. However for the non-abelian delta-functions this is not the case. For instance, in the case of $S U(2)$, we use the character expansion to obtain

$$
\delta(g)=\sum_{l} \sum_{m, n=-l}^{l} t_{m n}^{l}(g) \bar{t}_{m n}^{l}(1),
$$

where

$$
t_{m n}^{l}(g)=e^{i(m \phi+n \psi)} P_{m n}(\cos \theta),
$$

in terms of Euler angles. We see that only the $U(1)$ embeddings parametrized by the angles $\phi, \psi$ exponentiate in local form ( $g$ represents a local product of link variables around a plaquette). Because of the infinite product of Legendre polynomials we do not know how to perform the link integral (gauge field integral) and obtain a local model in the fields $l(z, \bar{z}), n(z, \bar{z}), m(z, \bar{z})$ defined on the dual lattice.

In the genus zero case and in the continuum we can circumvent the problems by using covariant Lagrange multipliers as we did previously. This allows us to project into the space of flat connections, however the problem reappears with the homotopy Wilson loops in the higher genus case, or even at tree level in the presence of operator insertions. These are serious problems in order to state nonabelian duality as a full-fledged string symmetry. In the particular case of genus 
one one can go a little further by using the fact that the fundamental group is abelian and flat connections can therefore be gauge transformed into the Cartan subalgebra of the group, thus allowing a treatment similar to the abelian case. When one tries to carry in detail the computations for genus higher than zero, one finds that the basic difficulty is the fact that the Hodge decomposition and the splitting of the flat connection part of the auxiliary gauge field only coincide for the abelian case. In some cases however one can study in some detail the global topological properties of the dual model directly.

The same type of problems appear when we try to carry out in detail the operator mapping between a given theory and its non-abelian dual. For instance, for a $\sigma$-model on a group manifold $G$, we could consider correlators of the form

$$
\left\langle\prod_{j} g\left(z_{j}, \bar{z}_{j}\right)\right\rangle,
$$

which can be expressed in the first order formalism by choosing a point $P=\infty$ and a system of cuts $\gamma_{j}$ going from it to $z_{j}$. Fixing $g(\infty)=1$ we can write

$$
\left\langle\prod_{j} g\left(z_{j}, \bar{z}_{j}\right)\right\rangle=\left\langle\prod_{j} W_{\gamma_{j}}\left(g^{-1} d g\right)\right\rangle,
$$

for

$$
W_{\gamma_{j}}\left(g^{-1} d g\right)=P e^{\int_{\gamma_{j}} g^{-1} d g},
$$

and in the gauge model we have

$$
\left\langle\prod_{j} g\left(z_{j}, \bar{z}_{j}\right)\right\rangle=\int \frac{D g D A D \chi}{|G|} e^{-S(g, A, \chi)} \prod_{j} W_{\gamma_{j}},
$$

with

$$
\begin{gathered}
W_{\gamma_{j}}=P e^{\int_{\gamma_{j}} g^{-1} D g} \\
D g=d g+A g,
\end{gathered}
$$

in the case that the isometries act by left multiplication. As usual we can take these 
operators as duals to the $g\left(z_{i}\right)$ but still we cannot perform the gauge field integration and express them in terms of the Lagrange multipliers due to the presence of the path ordering prescription.

It is quite clear that more work is needed to elucidate the complete structure of the non-abelian duality transformation.

Acknowledgements. We would like to thank X. De La Ossa, M.A.R. Osorio, F. Quevedo and M.A. Vázquez-Mozo for very useful discussions. E. Alvarez is grateful to P. Minkowski, P. Hajicek and other members of the ITP in Bern Universität for their hospitality while most of this work was done. His work was supported in part by the Tomalla Foundation (Switzerland) and by CICyT (Spain), contract number AEN/93/673. J.L.F. Barbón and Y. Lozano would like to thank the Theory Division at CERN for its hospitality while part of this work was done. J.L.F.B. was supported in part by a Fellowship from M.E.C. (Spain). Y.L. was supported in part by a Fellowship from Comunidad de Madrid ( Spain). 


\section{APPENDIX}

The vectorially gauged action reads:

$$
\begin{gathered}
S(g, A, \chi)=S(g)+\frac{k}{2 \pi} \int \operatorname{Tr}\left(A \bar{\partial} g g^{-1}-\bar{A} g^{-1} \partial g-g^{-1} A g \bar{A}+\right. \\
A \bar{A}+\chi(\partial \bar{A}-\bar{\partial} A+[A, \bar{A}]))
\end{gathered}
$$

(A.1) is invariant under local gauge transformations:

$$
g \rightarrow h^{-1} g h ; \quad A \rightarrow h^{-1}(A+d) h ; \quad \chi \rightarrow h^{-1} \chi h
$$

with $h \in S L(2, R)$. To gauge-fix this action we must choose a representative for every value of the trace and then fix the residual $U(1)$-symmetry on the lagrange multipliers.

Integration over the gauge fields yields the following result for the elliptic, hyperbolic and parabolic regions of $S L(2, R)$ :

Elliptic region:

$$
\begin{aligned}
& \tilde{S}=\frac{k}{2 \pi} \int \frac{1}{\rho^{2} \sin ^{2} \theta}\left[\rho^{2} \partial \rho \bar{\partial} \rho-\rho\left(\chi+\frac{1}{2} \sin 2 \theta\right)(\partial \rho \bar{\partial} \chi+\bar{\partial} \rho \partial \chi)-\right. \\
& \rho\left(\chi+\frac{1}{2} \sin 2 \theta\right)(\partial \rho \bar{\partial} \theta+\bar{\partial} \rho \partial \theta)+\left(\chi^{2}+\chi \sin 2 \theta+\sin ^{2} \theta\right) \partial \chi \bar{\partial} \chi+ \\
& \left.\left(\chi^{2}+\chi \sin 2 \theta+\sin ^{2} \theta\right)(\partial \chi \bar{\partial} \theta+\bar{\partial} \chi \partial \theta)+\left(\chi^{2}+\chi \sin 2 \theta+\left(1-\rho^{2}\right) \sin ^{2} \theta\right) \partial \theta \bar{\partial} \theta\right] \\
& \theta \in[0,2 \pi] ; \quad \rho, \chi \in R
\end{aligned}
$$

Hyperbolic region:

$$
\begin{aligned}
& \tilde{S}= \pm \frac{k}{2 \pi} \int \frac{1}{\rho^{2} \sinh ^{2} t}\left[\rho^{2} \partial \rho \bar{\partial} \rho \mp \rho\left(\chi+\frac{1}{2} \sinh 2 t\right)(\partial \rho \bar{\partial} \chi+\bar{\partial} \rho \partial \chi)\right. \\
& \quad \mp \rho\left(\chi+\frac{1}{2} \sinh 2 t\right)(\partial \rho \bar{\partial} t+\bar{\partial} \rho \partial t)+\left(\chi^{2}+\chi \sinh 2 t+\sinh ^{2} t\right) \partial \chi \bar{\partial} \chi+ \\
& \left.\quad\left(\chi^{2}+\chi \sinh 2 t+\sinh ^{2} t\right)(\partial \chi \bar{\partial} t+\bar{\partial} \chi \partial t)+\left(\chi^{2}+\chi \sinh 2 t+\left(1 \pm \rho^{2}\right) \sinh ^{2} t\right) \partial t \bar{\partial} t\right] \\
& \quad t, \rho, \chi \in R
\end{aligned}
$$


Parabolic region:

$$
\begin{aligned}
& \tilde{S}=\frac{k}{2 \pi} \int \frac{1}{x^{2}}[\partial x \bar{\partial} x+\partial y \bar{\partial} y \pm(\partial x \bar{\partial} y+\partial y \bar{\partial} x)] \\
& x, y \in R
\end{aligned}
$$

The new dilatons are:

$$
\begin{aligned}
& \tilde{\phi}=\phi-\log \left(8 \rho^{2} \sin ^{2} \theta\right) \\
& \tilde{\phi}=\phi-\log \left(8 \rho^{2} \sinh ^{2} t\right) \\
& \tilde{\phi}=\phi-\log \left(2 x^{2}\right)
\end{aligned}
$$

The singular points in each region correspond to the fixed points of the vectorial action. The dual metric in the parabolic region is singular, which means that in this region there is only one propagating degree of freedom. Since the parabolic region has zero measure in the whole dual space-time, this loss of degrees of freedom is possibly irrelevant.

The disconnectedness of the three regions makes unclear the interpretation of the dual space-time. One could also choose a different gauge fixing which does not distinguish between the three different regions in $S L(2, R)$, however one finds the same problem later when fixing the residual gauge symmetry action on the Lagrange multipliers. A possible way to treat the dual theory could be without eliminating the gauge-fields, using BRST techniques. 


\section{REFERENCES}

1. J.M. Drouffe and C. Itzykson: Quantum Field Theory and Statistical Mechanics, Cambridge U. Press, 1990.

2. L. Brink, M.B. Green, J.H. Schwarz: Nucl. Phys. B198 (1982), 474.

3. K. Kikkawa and M. Yamasaki: Phys. Lett. B149 (1984), 357 ; N. Sakai and I. Senda: Prog. Theor. Phys. 75 (1984), 692.

4. E. Alvarez and M.A.R. Osorio: Phys. Rev. D40 (1989), 1150.

5. K. Narain, H. Sarmadi and E. Witten: Nucl. Phys. B279 (1987), 369.

6. V. P. Nair, A. Shapere, A. Strominger and F. Wilczek: Nucl. Phys. B287 (1987), 402; A. Shapere and F. Wilczek: Nucl. Phys. B320 (1989), 609; A. Giveon, E. Rabinovici and G. Veneziano: Nucl. Phys. B322 (1989), 167.

7. T. H. Buscher: Phys. Lett. B194 (1987), 51 ; Phys. Lett. B201 (1988), 466.

8. A. Giveon, M. Roček: Nucl. Phys. B380 (1992), 128.

9. E. Kiritsis, Exact Duality Symmetry in CFT and String Theory, CERN-TH$6797 / 93$.

10. A. Font, L.E. Ibañez, D. Lüst and F. Quevedo: Phys. Lett. B245 (1990), 401; S.Ferrara, N. Magnoli, T.R. Taylor and G. Veneziano: Phys. Lett. B245 (1990), 409; H.P. Nilles and M. Olechowski: Phys. Lett. B248 (1990), 268;

P. Binetruy and M.K. Gaillard: Phys. Lett. B253 (1991), 119.

11. M. Roček and E. Verlinde: Nucl. Phys. B373 (1992), 630.

12. X.C. de la Ossa and F. Quevedo: Nucl. Phys. B403 (1993), 377.

13. I. Jack, D.R.T. Jones, N. Mohammedi and H. Osborn: Nucl. Phys. B332 (1990), 359; C. Hull and B. Spence: Phys. Lett. B232 (1989), 204.

14. S. Helgasson: Groups and Geometric Analysis, Academic Press, 1984.

15. E. Witten: Comm. Math. Phys. 92 (1984), 455. 
16. A.M. Polyakov and P.B. Wiegmann: Phys. Lett. 131B (1983), 121.

17. G.T. Horowitz and D.L. Welch: Phys. Rev. Lett. 71 (1993), 328.

18. G. Moore: Nucl. Phys. B293 (1987), 139; E. Alvarez and M.A.R. Osorio: Z. Phys. C44 (1989), 89.

19. E. Witten: Phys. Rev. D44 (1991), 314.

20. M. Bañados, M. Henneaux, C. Teitelboim and J. Zanelli: Geometry of the 2+1 Black Hole. To appear in Phys. Rev. D. 